OPEN ACCESS

Edited by:

Wei Tao,

Harvard Medical School,

United States

Reviewed by:

Yanqi Ye,

University of North Carolina at Chapel Hill, United States

Rongxin Su,

Tianjin University, China Bailiang Wang,

Wenzhou Medical University, China

*Correspondence:

Jianmeng Wang

jmwang1981@126.com

${ }^{\dagger}$ These authors have contributed equally to this work

Specialty section: This article was submitted to Experimental Pharmacology and Drug Discovery,

a section of the journal

Frontiers in Pharmacology

Received: 30 October 2018

Accepted: 14 January 2019

Published: 04 February 2019

Citation:

Niu K, Li N, Yao Y, Guo C, Ge Y and Wang J (2019) Polypeptide Nanogels With Different Functional Cores Promote Chemotherapy of Lung Carcinoma.

Front. Pharmacol. 10:37. doi: 10.3389/fphar.2019.00037

\section{Polypeptide Nanogels With Different Functional Cores Promote Chemotherapy of Lung Carcinoma}

\author{
Kai Niu' ${ }^{1 \dagger}$, Nan $\mathrm{Li}^{2 \dagger}$, Yunming $\mathrm{YaO}^{3 \dagger}$, Chunjie Guo ${ }^{4}$, Yuanyuan $\mathrm{Ge}^{5}$ and Jianmeng Wang ${ }^{5 *}$ \\ ${ }^{1}$ Department of Otorhinolaryngology Head and Neck Surgery, The First Hospital of Jilin University, Changchun, China, \\ ${ }^{2}$ Department of Neonatology, The First Hospital of Jilin University, Changchun, China, ${ }^{3}$ Department of Abdominal \\ Ultrasound, The First Hospital of Jilin University, Changchun, China, ${ }^{4}$ Department of Radiology, The First Hospital of Jilin \\ University, Changchun, China, ${ }^{5}$ Department of Geriatrics, The First Hospital of Jilin University, Changchun, China
}

Two kinds of tumor microenvironment-responsive polypeptide nanogels were developed for intracellular delivery of cytotoxics to enhance the antitumor efficacies and reduce the side effects in the chemotherapy of lung carcinoma. The sizes of both doxorubicin (DOX)-loaded nanogels methoxy poly(ethylene glycol)-poly(Lphenylalanine-co-L-cystine) [mPEG-P(LP-CO-LC)] and methoxy poly(ethylene glycol)poly(L-glutamic acid-co-L-cystine) [mPEG-P(LG-Co-LC)] (NGP/DOX and NGG/DOX) were less than $100 \mathrm{~nm}$, which was appropriate for the enhanced permeability and retention (EPR) effect. The bigger and smaller scale of nanoparticle could induce the elimination of reticuloendothelial system (RES) and decrease the in vivo circulating halflife, respectively. The loading nanogels were stable in the neutral environment while quickly degraded in the mimic intracellular microenvironment. Furthermore, the DOXloaded reduction-responsive nanogels showed significantly higher tumor cell uptake than free DOX. $\mathrm{HCl}$ as time went on from 2 to $6 \mathrm{~h}$. In addition, these DOX-loaded nanogels showed efficient antitumor effects in vivo, which was verified by the obviously increased necrosis areas in the tumor tissues. Furthermore, these DOX-loaded nanogels efficiently reduced the side effects of DOX. In conclusion, these reduction-responsive polypeptides based nanogels are suitable for the efficient therapy of lung carcinoma.

Keywords: nanogel, reduction-responsive, controlled drug delivery, lung carcinoma, antitumor

\section{INTRODUCTION}

Lung carcinoma has been the most common malignancy in both men and women with high mortality (Kanodra et al., 2015; Kernstine, 2017). Chemotherapy, as a traditional treatment, is important to the treatment of lung cancer. However, the main therapeutic platforms available for chemotherapy drugs currently have the disadvantages of not being able to effectively aggregate in tumor cells and causing various systemic side effects. Due to these deficiencies, various nanomedicine delivery systems have been studied such as micelles (Feng et al., 2017; Shen et al., 2017; Xu et al., 2017; Kosakowska et al., 2018; Li et al., 2018b; Sun et al., 2018), nanogels (Ding et al., 2011; Guo et al., 2017, 2018; Jiang et al., 2018b; Li et al., 2018d; Zhang et al., 2018; Zhu et al., 2018), polymer-drug conjugates (Zhao et al., 2017; Cong et al., 2018; Li et al., 2018a; Yang et al., 2018), liposomes (Allen and Cullis, 2013; Zhao et al., 2013; Piffoux et al., 2018), and so forth 
(Ding et al., 2014; Tao et al., 2015, 2017; Chen et al., 2017a; Armstrong and Stevens, 2018; Ji et al., 2018; Jiang et al., 2018a; Li et al., 2018b,c; Xiao et al., 2018), which help to improve the drug accumulation in the tumor, to achieve the effective control of drug release in the tumor lesions, and to reduce the side effects (Yu et al., 2016; Zhang et al., 2017). Among them, nanogels have the strong core-shell structures by crosslinking, which enables them not only to exhibit high drug loading capacity, but also to prevent drug leakage (Ye et al., 2016; Chen et al., 2017b). In addition, the suitable sizes of the nanogels made them can efficiently accumulate at the tumor lesions by the enhanced permeability and retention (EPR) effect (Acharya and Sahoo, 2011). More importantly, additional functionality is given to crosslinkers to achieve "switch on/off" release of drugs from nanogels in tumor cells (Mura et al., 2013). In addition, the stability of nanogels is one of the major obstacles, which handers the in vivo application of them. Fortunately, the chemistry crosslinking has been widely used to enhance the stability and functionality of nanogels, which is more stable than non-covalent interactions (Ryu et al., 2010; Jiang et al., 2018a). Furthermore, cross-linking nanoparticles with different functional chemical crosslinkers could controllably release the laden drugs according to the redox, low $\mathrm{pH}$, and high enzyme level of tumor microenvironments (Zha et al., 2011).

Due to the different metabolic pathways of tumor cells, the microenvironments of tumor tissues show hypoxia, low sugar, and low pH (Parks et al., 2013). It is worth noting that malignant cells show the reductive intracellular microenvironment (Ge and Liu, 2013). Therefore, the reduction-sensitive polymeric nanoparticles have attracted more and more attention in the realm of smart antitumor drug delivery (Cheng et al., 2013; Phillips and Gibson, 2014).

Herein, we reported the drug delivery potential of reductionsensitive polypeptide nanogels formulations, which could suppress lung carcinoma cell proliferation at low dose and reduce unwanted adverse effects. The reduction-responsive methoxy poly(ethylene glycol)-poly(L-phenylalanine-co-Lcystine) (mPEG-P(LP-co-LC)) and methoxy poly(ethylene glycol)-poly(L-glutamic acid-co-L-cystine) mPEG-P(LG-co-LC) nanogels were prepared to selectively deliver chemotherapy agents (Scheme 1). Typically, nanogels loaded with doxorubicin (DOX) were used as models for clinical antitumor drug. Results showed that both DOX-loaded nanogels exhibited satisfactory antitumor activity and higher safety than free $\mathrm{DOX} \cdot \mathrm{HCl}$. These DOX-loaded nanogels are able to serve as satisfactory nanoplatforms for the therapy of lung carcinoma.

\section{MATERIALS AND METHODS}

Methoxy (ethylene glycol) (mPEG) was purchased from Aladdin Industrial Co., Ltd. (Shanghai, China) and mPEG-NH $\mathrm{N}_{2}$ was synthesized by trimethylamine modified. L-Glutamic acid (LC), L-phenylalanine (LP), and L-cystine (LC) were obtained from GL Biochem, Ltd. (Shanghai, China). LG NCA, LP NCA, and LC NCA were prepared as reported in previous work (Huang et al., 2015; Shi et al., 2017). Doxorubicin hydrochloride (DOX.HCl) was bought from Beijing Huafeng United Technology Co., Ltd. (Beijing, China). Glutathione (GSH) (used for cell culture) was bought from Aladdin Reagent Co., Ltd. (Shanghai, China). Both 4,6-Diamidino-2-phenylindole (DAPI) and methyl thiazolyl tetrazolium (MTT) were obtained from Sigma-Aldrich (Shanghai, China). Hematoxylin and eosin were purchased from Merck Company (Darmstadt, Germany).

\section{Characterizations}

Proton Nuclear Magnetic Resonance $\left({ }^{1} \mathrm{H}\right.$ NMR) spectra were detected on a Bruker AV 600 NMR spectrometer (Billerica, MA, United States) using deuterated dimethyl sulfoxide (DMSO- $d_{6}$ ) as the solvent. Fourier-transform infrared spectroscopy (FTIR) was performed on a Bio-Rad Win-IR instrument (Bio-Rad Laboratories Inc., Cambridge, MA, United States) by using potassium bromide method. The morphology of NGP/DOX and NGG/DOX nanogels were visualized on JEM-1011 transmission electron microscope (TEM; JEOL, Tokyo, Japan). Sizes of NGP/DOX and NGG/DOX were determined by dynamic laser scattering (DLS) measurement on a WyattQELS instrument (DAWN EOS, Wyatt Technology Corporation, Santa Barbara, CA, United States), and the scattering angle was set at $90^{\circ}$.

\section{Syntheses and Characterizations of MPEG-P(LP-co-LC) and MPEG-P(LG-Co-LC)}

The reduction-responsive $\mathrm{mPEG}-\mathrm{P}(\mathrm{LP}-\mathrm{co}-\mathrm{LC})$ nanogel was synthesized through the one-step ROP of LP NCA and LC NCA with amino-terminated $\mathrm{mPEG}\left(\mathrm{mPEG}-\mathrm{NH}_{2}\right)$ as a macroinitiator according to the reports in our previous works. Firstly, $2 \mathrm{~g}$ of mPEG-NH $H_{2}, 2.3 \mathrm{~g}$ of NCA LP NCA and $0.9 \mathrm{~g}$ of LC NCA were dissolved in $100 \mathrm{~mL}$ of DMF and stirred for three days. The obtained solution was poured into $700 \mathrm{~mL}$ of the ethyl ether twice, and the white precipitate was collected. After vacuum drying, mPEG-P(LP-co-LC) was obtained with the yield of 75.2\%. mPEG-P(BLG-co-LC) was prepared in a similar route. $\mathrm{mPEG}-\mathrm{P}(\mathrm{LG}-\mathrm{co}$-LC) was generated by removing the benzyl group from mPEG-P(BLG-co-LC). mPEG-P(BLG-co-LC) was dissolved in dichloroacetic acid (100 $\mathrm{mg} \mathrm{mL}^{-1}$ ) and a 33 wt. \% solution of $\mathrm{HBr}$ in acetic acid was added subsequently $(20 \mathrm{~mL}$ for $1 \mathrm{~g}$ copolymer). After stirring for $1 \mathrm{~h}$ at $30^{\circ} \mathrm{C}$, the mixture was precipitated into diethyl ether (10 times volume of the reaction solution). The obtained product was further dried under vacuum at room temperature for $24 \mathrm{~h}$ after washing twice with diethyl ether (Yield: $80.5 \%$ ). The products were characterized by proton nuclear magnetic resonance $\left({ }^{1} \mathrm{H}\right.$ NMR), Fouriertransform infrared (FT-IR), inductively coupled plasma optical emission spectroscopy (ICP-OES), and elemental analysis.

\section{DOX Encapsulation}

Nanogel was loaded with DOX by nanoprecipitation method. Firstly, $50 \mathrm{mg}$ of nanogel was dispersed in $20 \mathrm{~mL}$ of N,Ndimethylformamide (DMF). After adding $10 \mathrm{mg}$ of $\mathrm{DOX} \cdot \mathrm{HCl}$, the solution was further stirred for $12 \mathrm{~h}$ at room temperature. Then, $2 \mathrm{~mL}$ of phosphate buffered saline (PBS; $0.01 \mathrm{M}$ ) was slowly mixed into the above solution, along with $18 \mathrm{~mL}$ of MilliQ water. 


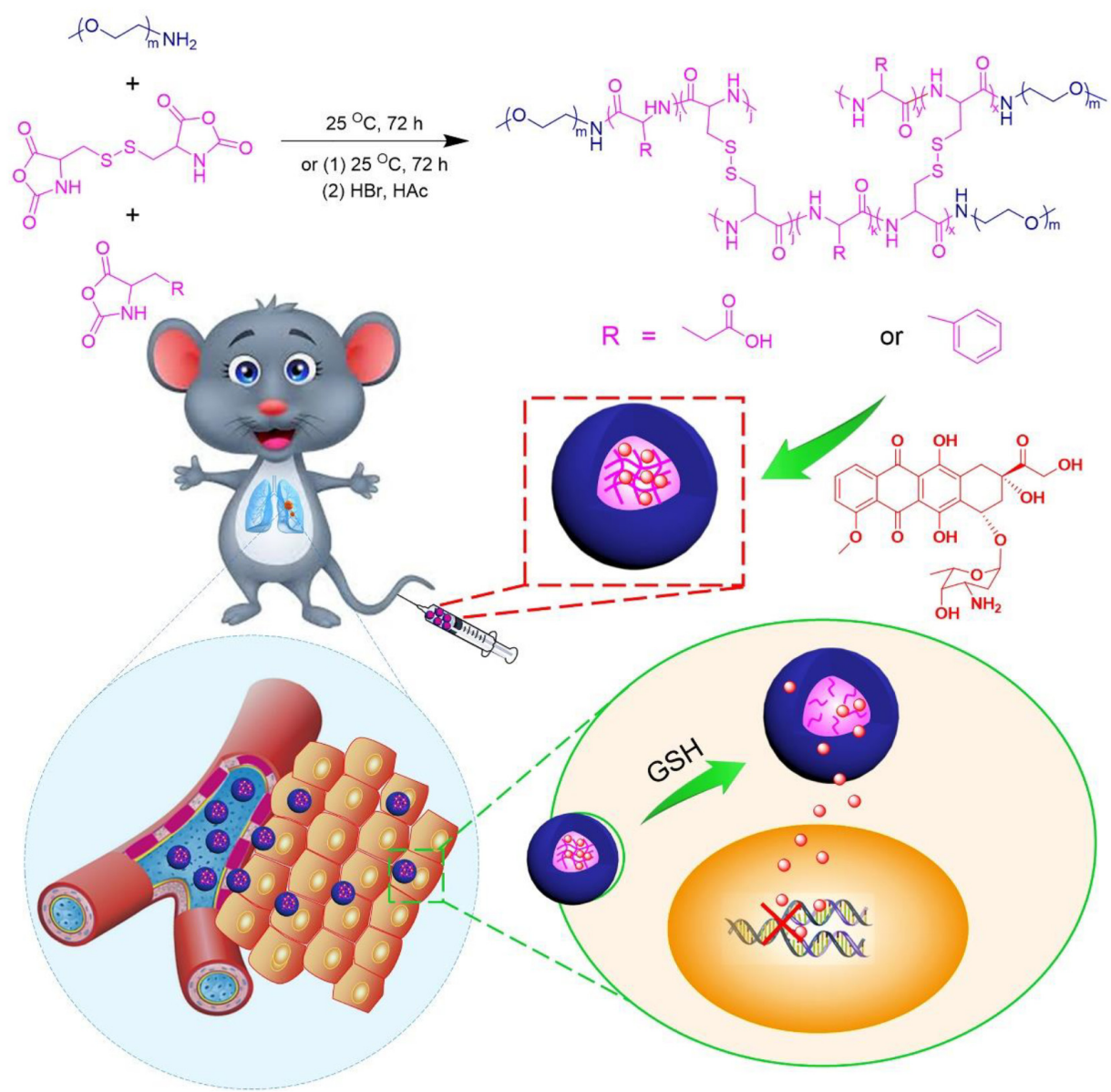

SCHEME 1 | Synthetic pathway for mPEG-P(LP-CO-LC) and mPEG-P(LG-CO-LC) nanogel, illustrations of DOX encapsulation by nanogel, and its circulation, intratumoral accumulation, endocytosis, and targeting intracellular DOX release after intravenous injection.

The final mixture was dialyzed against MilliQ water for $24 \mathrm{~h}$ (molecular weight cut-off $(\mathrm{MWCO})=3500 \mathrm{Da}$ ) after stirring for $12 \mathrm{~h}$ at room temperature. The MilliQ water was changed every 2 h. Finally, NGP/DOX and NGG/DOX nanogels were obtained by filtration and lyophilization.

The drug loading content (DLC) and drug loading efficiency (DLE) were detected by standard curve method, using fluorescence spectroscopy on a Photon Technology International (PTI) Fluorescence Master System with Felix 4.1.0 software (PTI, Lawrenceville, NJ, United States; kex $=480 \mathrm{~nm})$. The DLC and DLE of NGP/DOX and NG/DOX were calculated according to Eqs. (1) and (2), respectively.

$$
\begin{gathered}
\text { DLC }(\%)=\frac{\text { Weight of Drug in Nanogel }}{\text { Weight of Drug - Loaded Nanogel }} \times 100 \% \\
\text { DLE }(\%)=\frac{\text { Weight of Drug in Nanogel }}{\text { Weight of Feeding Drug }} \times 100 \%
\end{gathered}
$$

\section{In vitro DOX Release}

In vitro drug release profiles of DOX from NGP/DOX and NGG/DOX nanogels were performed in PBS ( $\mathrm{pH} 7.4$ ) with or without $10 \mathrm{nM}$ GSH. $10 \mathrm{~mL}$ of nanogel $\left(0.1 \mathrm{mg} \mathrm{mL} \mathrm{mL}^{-1}\right)$ aqueous solution was transferred into an end-sealed dialysis bag $(\mathrm{MWCO}=3500 \mathrm{Da})$. The release experiment was carried out by putting the end-sealed dialysis bag into the corresponding release medium $(100 \mathrm{~mL})$ at $37^{\circ} \mathrm{C}$ with continuous shaking at $75 \mathrm{rpm}$ in the dark. At fixed time intervals, $2 \mathrm{~mL}$ of release medium was removed and an equal volume of fresh medium was added. The amount of released DOX was measured by the fluorescence spectrophotometer $(\mathrm{kex}=480 \mathrm{~nm})$.

\section{Cell Culture}

Under the conditions of $37^{\circ} \mathrm{C}$ and $5 \%(\mathrm{~V} / \mathrm{V})$ carbon dioxide $\left(\mathrm{CO}_{2}\right)$, the human lung Lewis cells were cultured in RPMI1640 , which was supplemented with $10 \%$ (V/V) fetal bovine serum (FBS), penicillin (100 IU $\left.\mathrm{mL}^{-1}\right)$, and streptomycin (100 IU $\left.\mathrm{mL}^{-1}\right)$.

\section{Intracellular DOX Release}

The intracellular DOX release from NGP/DOX and NGG/DOX were measured by confocal laser scanning microscopy (CLSM) toward Lewis cells. The cells (15,000 cells) were seeded in disks, 
incubated in $1 \mathrm{~mL}$ of RPMI-1640 medium containing $10 \%$ FBS for $24 \mathrm{~h}$, and pretreated with $10 \mathrm{mM}$ GSH or buthionine sulfoximine (BSO) for $2 \mathrm{~h}$. After removing the medium and subsequently washing three times with PBS ( $\mathrm{pH}$ 7.4) solution, $1 \mathrm{~mL}$ of NGP/DOX and NGG/DOX solution in RPMI-1640 was added, with a final DOX dose of $10 \mu \mathrm{g} \mathrm{mL}^{-1}$. The cells treated with equivalent free DOX without GSH pretreatment were used as control. After another $2 \mathrm{~h}$ of incubation, the cells were washed with PBS for five times, and fixed with $4 \%$ $(\mathrm{W} / \mathrm{V})$ PBS-buffered paraformaldehyde at room temperature for $30 \mathrm{~min}$. The cellular nuclei were then stained at $37^{\circ} \mathrm{C}$ for 3 min using DAPI. A CLSM (Carl Zeiss, LSM 780, Jena, Germany) was used to view the intracellular localization of DOX.

\section{Cytotoxicity Assays}

The cytotoxicities of NGP/DOX, NGG/DOX and free DOX were evaluate din Lewis cells at different conditions. The cells were planted in 96-well plates $\left(7 \times 10^{3}\right.$ cells per well $)$ in $200 \mu \mathrm{L}$ of RPMI-1640 medium supplemented with 1X penicillin/streptomycin and $10 \%$ fetal bovine serum. After incubation for $24 \mathrm{~h}$ at $37^{\circ} \mathrm{C}$, the cells were pretreated with $10 \mathrm{mM} \mathrm{GSH}$ or BSO for $2 \mathrm{~h}$. Subsequently, each culture medium was replaced by $180 \mu \mathrm{L}$ of RPMI-1640 containing NGP/DOX, NGG/DOX and free DOX at equivalent concentrations, respectively, with the DOX ranging from 0.3 to $18.4 \mu \mathrm{M}$. After a further 24,48 , and $72 \mathrm{~h}$ incubation, $20 \mu \mathrm{L}$ MTT (5 mg mL ${ }^{-1}$ ) in PBS was added to each well, followed by another $4^{\circ} \mathrm{h}$ incubation at $37^{\circ} \mathrm{C}$. Then the sediment was dissolved in $150 \mu \mathrm{L}$ DMSO after the medium was removed. The absorbance at $490 \mathrm{~nm}$ of the above solution was determined on an ELx808 microplate reader (Bio-Tek Instruments, Inc., Winooski, VT, United States). The percentage of cell viability was determined by comparing the absorbance of the sample cells and the control cells [Eq. (3)].

$$
\text { Cell }(\%)=\frac{A_{\text {Sample }}}{A_{\text {Control }}} \times 100 \%
$$

\section{Animal Procedures}

5-Week-old male BALB/c mice weighting $18 \pm 0.2 \mathrm{~g}$ were supplied by the Jilin University Experiment Animal Center (Changchun, China). All animal experiments were performed according to the Guidelines for Animal Care and Use of Jilin University. The tumor grafted mouse model was established by subcutaneous injection of $100 \mu \mathrm{L}$ of cell suspension containing $2 \times 10^{6}$ Lewis cells in PBS into the armpit of right anterior forelimb.

\section{In vivo Antitumor Assessments}

The tumor volumes and mice's body weights were monitored every two days from the second day after the inoculation of Lewis cells (that was, Day 1). When tumor volume increased to about $100 \mathrm{~mm}^{3}$ after 8 days of inoculation, the nude mice were randomly divided into 7 groups $(n=10)$, that was, free DOX, NGP/DOX or NGG/DOX at a DOX dose of 3 or $6 \mathrm{mg}(\mathrm{kg} \mathrm{BW})^{-1}$ and normal saline (control group). The formulations of DOX were recorded as DOX/3, DOX/6, NGP/DOX/3, NGP/DOX/6, NGG/DOX/3 and NGG/DOX/6, respectively. At the same time, the treatments began with injecting $100 \mu \mathrm{L}$ of normal saline and various DOX preparations in normal saline into the tail vein of mice for four times every 5 days. The tumor sizes were measured every day, and the body weights were measured every subsequent day. Tumor volumes [Eq. (4)] and body weights were used to evaluate the antitumor efficacy and security in vivo.

$$
V\left(\mathrm{~mm}^{3}\right)=\frac{\mathrm{L} \times \mathrm{S}^{2}}{2}
$$

In Eq. (4), L ( $\mathrm{mm}$ ) was the largest diameter of tumor, and S (mm) was the smallest diameter.

The following formula was used to calculate the tumor inhibition ratio:

$$
\text { Tumor inhibition rate }(\%)=\left(V_{\text {control }}-V_{\text {sample }}\right) / V_{\text {control }}
$$

In Eq. (5), $V_{\text {control }}$ and $V_{\text {sample }}$ represented the tumor volumes of control groups and sample groups, respectively.

The weights of the major organs were recorded. The organ indices of all the organs of mice were calculated by [Eq. (6)].

$$
\operatorname{Organ}(\%)=\frac{W_{\text {Organ }}}{W_{\text {Body }}} \times 100 \%
$$

\section{Immunohistochemical Analyses of Tumor Tissues}

On day 27, the Lewis lung carcinoma-grafted BALB/c mice were killed by cervical dislocation 5 days after the last injections. The tumors and major organs (heart, liver, spleen, lung, kidney, thymus, and marrow) were isolated at first, and then fixed with $4 \%$ (W/V) paraformaldehyde overnight, followed by dehydration, clearing, wax infiltration, and embedding. The paraffin-embedded tumors and organ tissues were cut at a thickness of $5 \mu \mathrm{m}$ for hematoxylin and eosin (H\&E) staining. Paraffin sections with a thickness of $3 \mu \mathrm{m}$ were used for immunohistochemical staining (including caspase-3, survivin, $\mathrm{Bax}$, and $\mathrm{Bcl}-2$ ) to assess the pathological and immunological characteristics of tumor tissues. The instruments used included Leica RM 2245 paraffin machine (Leica, Germany), Leica HI1210 fishing machine (Leica, Germany), Leica EG1150H embedding machine (Leica, Germany), Leica HI1220 booth machine (Leica, Germany), Olympus BX51 microscope (Olympus, Japan), and Motic image analysis system (Motic Industrial Group Co., Ltd., Xiamen, China).

\section{Histopathological and Biochemical Analyses of Organs}

The major internal organs and tissues (heart, liver, spleen, lung, kidney, thymus, and marrow) were collected at the same time. The organs from healthy mice were used as controls. All the organs involved were divided into two parts as follows: (i) one part (excluding marrow) fixed with $4 \%$ $(W / V)$ PBS-buffered paraformaldehyde was prepared for the histopathological analyses by H\&E staining. (ii) The other 
part was used to detect the organ function-related biochemical indicators, including blood urea nitrogen (BUN), creatinine (Cr), alanine aminotransferase (ALT), aspartate aminotransferase (AST), creatine kinase (CK), creatine kinase-MB (CK-MB) and lactate dehydrogenase (LDH), by commercial enzyme-linked immunosorbent assay (ELISA) kits. The biochemical indicators in serum were also tested. Briefly, $300 \mu \mathrm{L}$ of blood without anticoagulant was centrifuged at $3000 \mathrm{rpm}$ for $10 \mathrm{~min}$. The serum was then collected to detect the clinical biochemical parameters. The data from healthy nude mice were used as controls. The histopathological results were detected and analyzed by Olympus BX51 microscope and Motic image analysis system, respectively.

\section{Detections of White Blood Cell (WBC) Count and Bone Marrow Cell Micronucleus Rates (BMMRs)}

On day 27, $20 \mu \mathrm{L}$ of blood (anticoagulated through enucleation method) was taken from each nude mouse to count the WBCs. The sternums from BALB/c mice were decalcified and fixed for 10 days after being placed in $10 \%(\mathrm{~V} / \mathrm{V})$ formic acid-formalin solution. The data from normal nude mice were used as controls. Then, the tissues were dehydrated, cleared, wax infiltrated, and embedded. For each sternum, paraffin sections with a thickness of $5 \mu \mathrm{m}$ were collected for $\mathrm{H} \& \mathrm{E}$ staining, with an interval of $50 \mu \mathrm{m}$. The H\&E-stained section was used to evaluate the BMMR.

\section{Statistical Analyses}

All tests were carried out at least three times, and the relevant data were expressed as mean \pm standard deviation (SD). Statistical analysis was performed using SPSS 13.0 statistical software (SPSS Inc., Chicago, IL, United States), ${ }^{* * *} P<0.05$ was considered statistically significant, and ${ }^{* *} P<0.01$ and ${ }^{*} P<0.001$ were as considered significant differences.

\section{RESULTS AND DISCUSSION}

\section{Characterizations of NGP/DOX and NGG/DOX}

These reduction-responsive nanogels consisted of $\mathrm{mPEG}$ (hydrophilic shell) and disulfide-cross-linked P(LP-co-LC) or $\mathrm{P}$ (LG-co-LC) (hydrophobic core). The disulfide bond of the LC segment endowed the nanogels with reduction-responsiveness (Huang et al., 2015; Shi et al., 2017). These DOX-loaded nanogels were prepared through a modified nanoprecipitation method
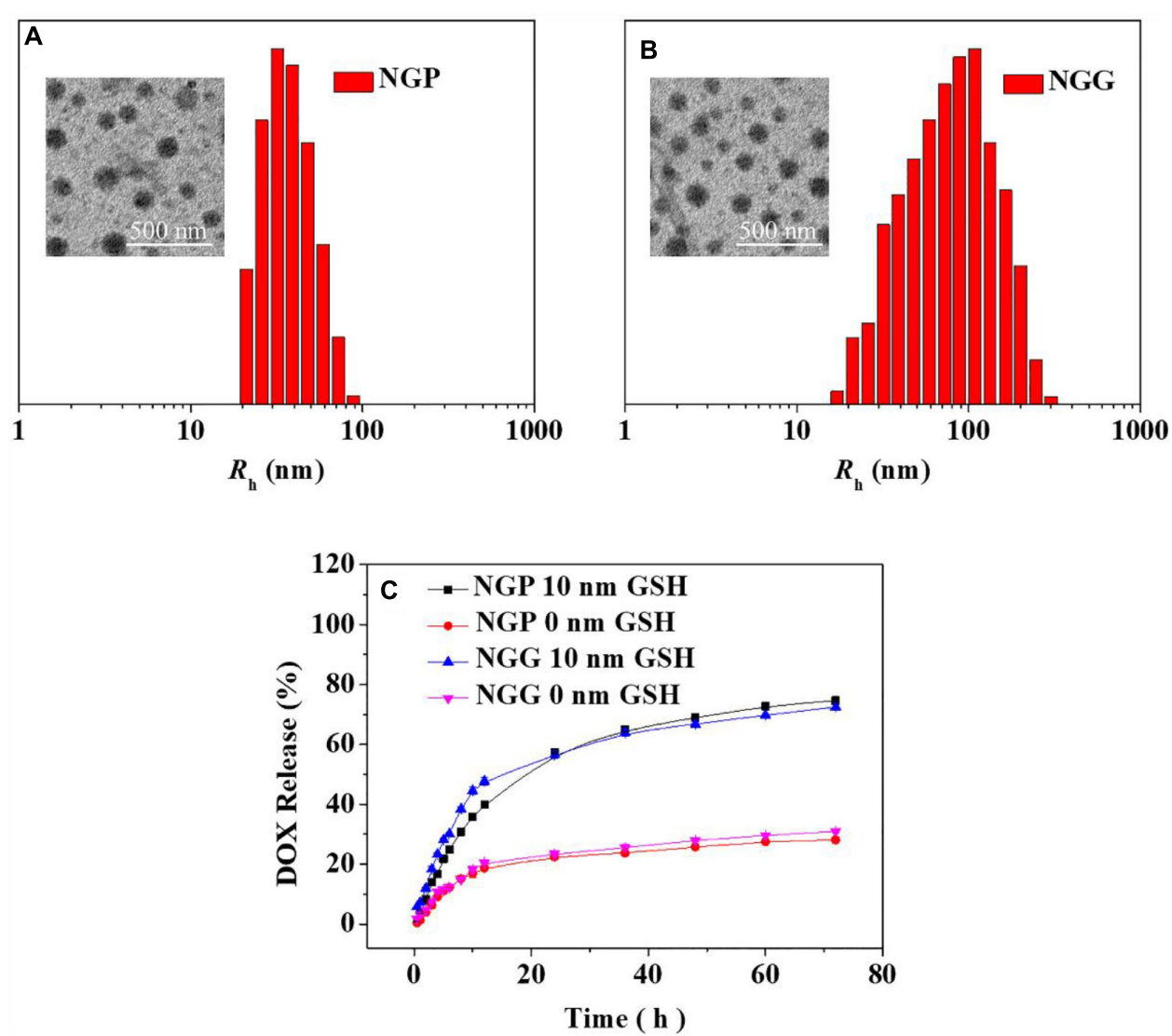

FIGURE 1 | Chemo-Physical Characterizations. Typical TEM micrograph and $R_{h}$ detected by DLS of NGP/DOX (A) and NGG/DOX (B). In vitro DOX release from NGP/DOX and NGG/DOX (C) in PBS of pH 7.4 and 7.4 with $10 \mathrm{~nm} \mathrm{GSH}$ at $37^{\circ} \mathrm{C}$. Scale bar in B represents $500 \mathrm{~nm}$. The statistical data are represented as mean \pm standard deviation $(S D ; n=3)$. 
by adding DOX aqueous solution to dimethylformamide (DMF) solution containing NGP or NGG nanogel (Scheme 1) (Ding et al., 2013a; Huang et al., 2015; Liu et al., 2015; Shi et al., 2017). The drug loading content (DLCs) and drug loading efficiency (DLEs) of NGP/DOX and NGG/DOX were calculated to be $9.8,54.7$ and $15.2,91.5$ wt.\%, respectively. As shown in the TEM images, NGP/DOX, and NGG/DOX showed a spherical morphology at a diameter of about 80 and $93 \mathrm{~nm}$, respectively (Figures 1A,B). The $R_{\mathrm{h}}$ of NGP/DOX and NGG/DOX detected by dynamic laser scattering (DLS) were $78.1 \pm 3.5$ and $93.3 \pm 3.5 \mathrm{~nm}$, respectively (Figures 1A,B). The hydrodynamic size of NGP/DOX and NGG/DOX was slightly larger than the diameter detected by TEM due to the swelling of nanogels in the aqueous condition (Huang et al., 2015; Shi et al., 2017). Appropriate size facilitated the efficient accumulation of nanogels in the tumors (Ding et al., 2013b,c, 2015).

\section{In vitro Release Performance and Tumor Cell Inhibition}

The DOX release performances of NGP/DOX and NGG/DOX were carried out in PBS with 0 or $10.0 \mathrm{mM}$ glutathione (GSH). As shown in Figure 1C, during the 72 h test, only around 30\%
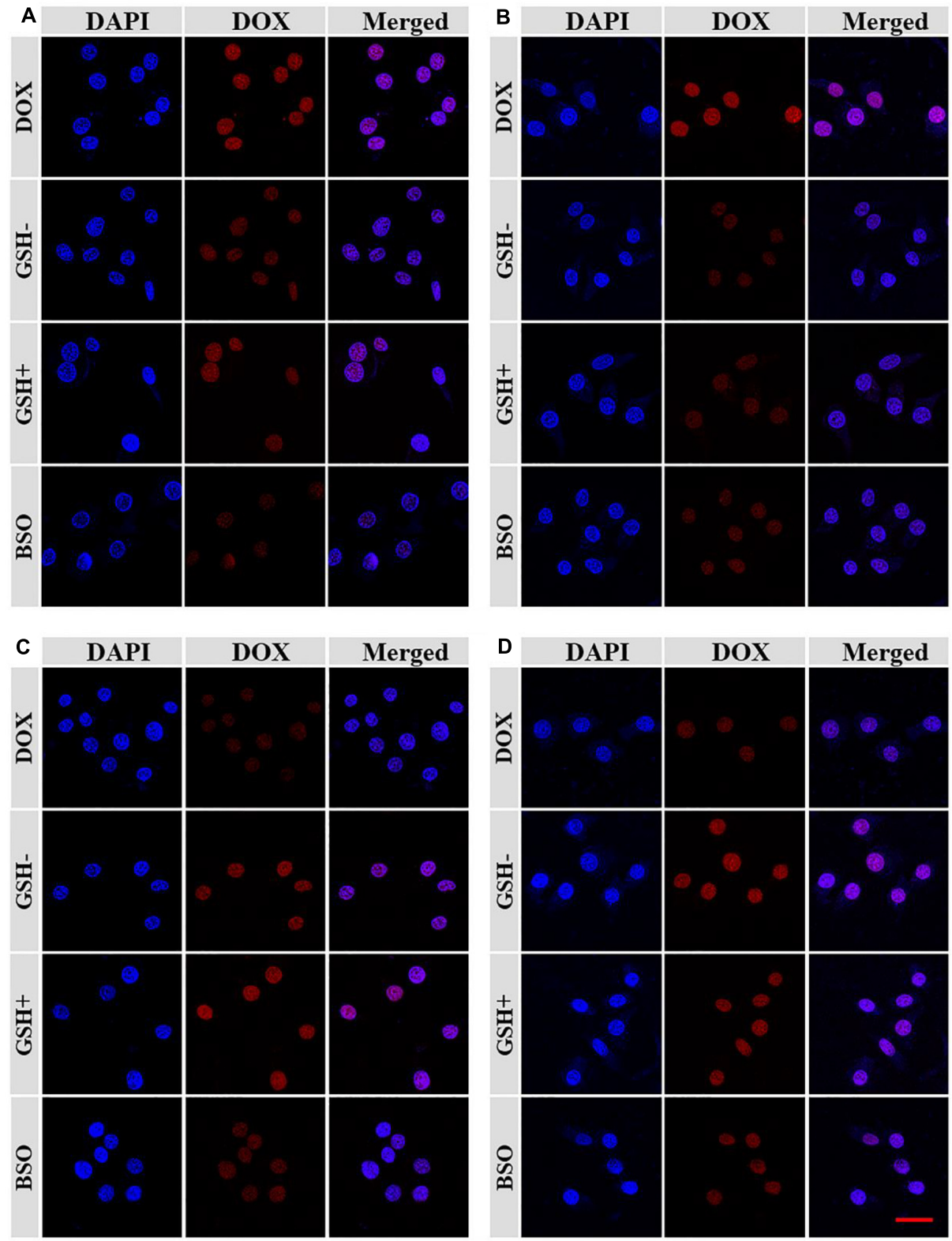

FIGURE 2 | The CLSM microimages analyses for cell internalization of NGP (A,C) and NGG (B,D) after incubation with Lewis cells for $2 \mathrm{~h}(\mathbf{A}, \mathbf{B})$ and $6 \mathrm{~h}$ (C,D). Scale bar $=50 \mu \mathrm{m}$. 
of DOX was released in the PBS with $0 \mathrm{mM}$ GSH. In contrast, an obviously increased DOX release was observed in the PBS with $10.0 \mathrm{mM}$ GSH. In detail, the proportions of cumulative released DOX from NGP/DOX and NGG/DOX in the initial $12 \mathrm{~h}$ were 47.8 and $39.8 \%$ in PBS with GSH (GSH+), respectively. After $72 \mathrm{~h}$ of incubation, the DOX released from NGP/DOX and NGG/DOX in the GSH+ medium (72.4 and 74.7\%) were more than twice that in the GSH- medium (31.0 and 28.1\%), respectively. The accelerated release of DOX should be due to GSH breaking the disulfide bond of nanogels (Huang et al., 2015). After $24 \mathrm{~h}$, the drug release of nanogels was relatively decelerated and sustained. The release results indicated the NGP/DOX and NGG/DOX could efficiently release the DOX in tumor cells according to the different redox potential between intracellular and extracellular microenvironments, which might have more obvious antitumor activity.

Confocal laser scanning microscopy (CLSM) assays were performed toward Lewis lung carcinoma cells pretreated without (GSH-), with $10.0 \mathrm{mM} \mathrm{GSH}(\mathrm{GSH}+)$ or buthionine sulfoximine (BSO) to verify the satisfactory intracellular DOX release of NGP/DOX and NGG/DOX. NG/DOX with a dosage of $10.0 \mu \mathrm{g}$ $\mathrm{mL}^{-1} \mathrm{DOX} \cdot \mathrm{HCl}$ was used to co-cultured with the GSH- or GSH+ cells for 2 and $6 \mathrm{~h}$. The unpretreated cells co-cultured with equivalent free $\mathrm{DOX} \cdot \mathrm{HCl}$ were prepared as a control. As shown, the DOX fluorescence was shown in all the Lewis lung carcinoma cells treated with DOX formulations (Figure 2). As
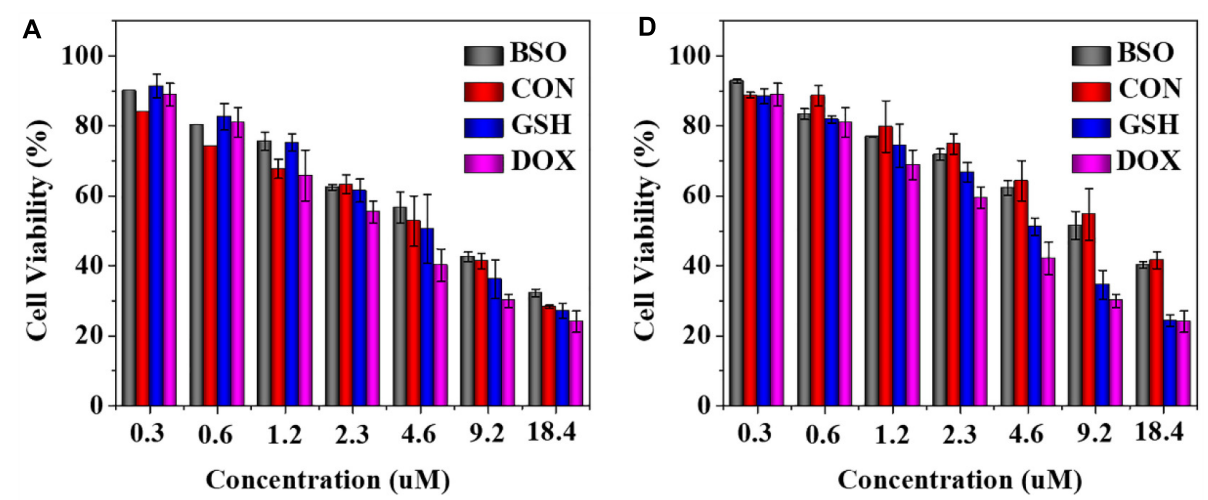

B

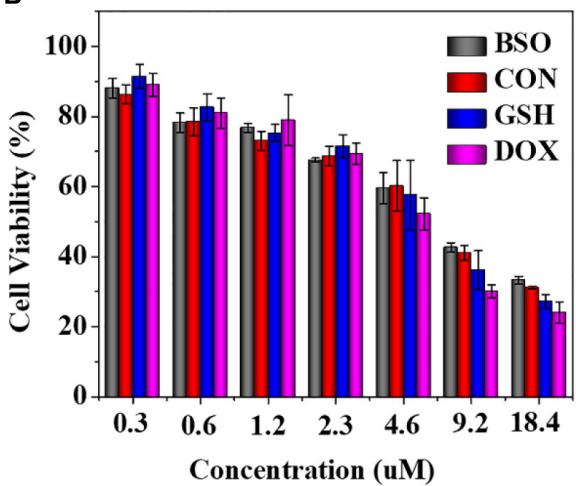

E
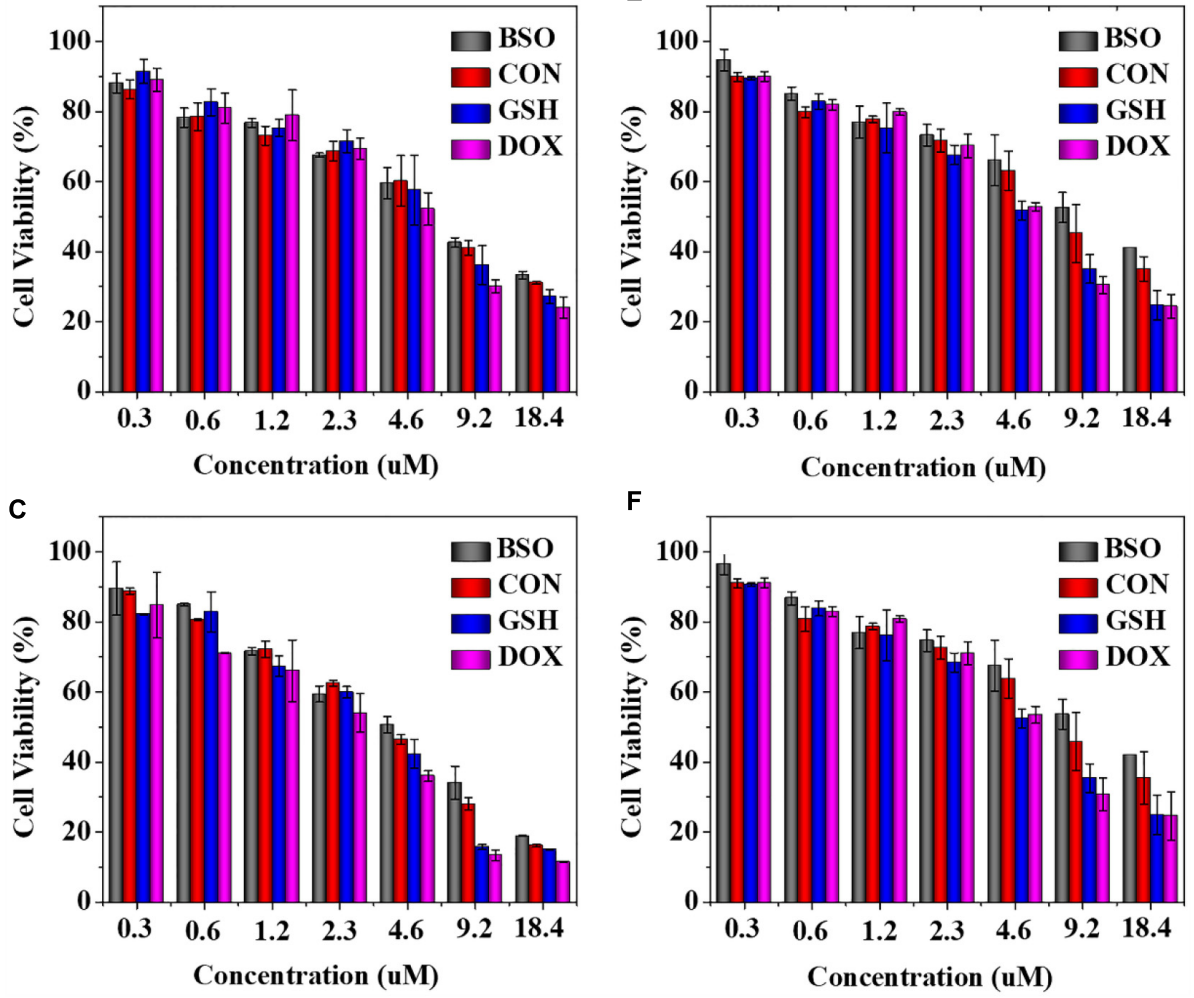

$\mathbf{F}$

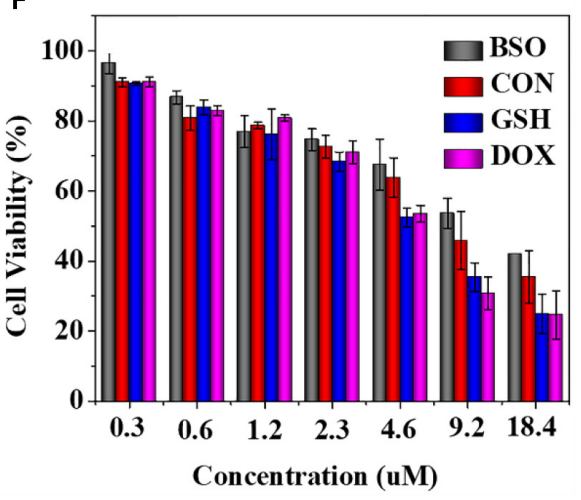

FIGURE 3 | In vitro cytotoxicity of NGP (A-C) and NGG (D-F) after incubation with Lewis cells for 24,48 , and $72 \mathrm{~h}$. The statistical data are presented as a mean $\pm S D(n=6)$. 
time went on, the DOX fluorescence in the nanogels with GSH pretreatment groups were higher than that of the free DOX.HCl and BSO pretreated groups. As far as we knew, only the DOX released from nanogels in the cells could be detected by CLSM (Ding et al., 2013c). These results verified the efficient endocytosis as well as the reduction-responsive intracellular DOX release of NGG/DOX and NGP/DOX. These results might be related to that with the pretreatment of GSH, the intracellular GSH content was increased, which facilitated the rapid release of DOX from nanogels. Moreover, the results were consistent with the in vitro DOX release kinetics in PBS (Figure 1C). It was interesting that the tumor cells incubated with free DOX. $\mathrm{HCl}$ exhibited the strongest DOX fluorescence at $2 \mathrm{~h}$. This result was related to the way of free DOX $\cdot \mathrm{HCl}$ entered cells was diffusion, which was faster than the endocytosis of nanogels (Ding et al., 2013b,c).

The in vitro antitumor activities of NGG/DOX and NGP/DOX were estimated by MTT assay at 24, 48, and $72 \mathrm{~h}$. As shown in Figure 3, compared with the BSO pretreated and unpretreated groups, GSH pretreated nanogels showed obviously higher growth inhibition efficiency in Lewis cells during the test time. This more satisfactory inhibition of GSH pretreated nanogels might be related to the intracellular GSH content was increased after GSH pretreatment, which facilitated the rapid release of DOX from nanogels and showed stronger tumor killing effect. As time went on, the half maximal inhibitory concentrations $\left(\mathrm{IC}_{50} \mathrm{~s}\right)$ of NGP/DOX decreased, but the order was constant: BSO $<\mathrm{GSH}-<\mathrm{GSH}+$. At $72 \mathrm{~h}$, the $\mathrm{IC}_{50}$ s of BSO, GSH-, and GSH+ pretreated groups were calculated to be 3.78, 3.30, and $2.61 \mu \mathrm{M} \mathrm{mL}^{-1}$, respectively. The NGG/DOX groups showed similar results, and the $\mathrm{IC}_{50} \mathrm{~s}$ of $\mathrm{BSO}, \mathrm{GSH}-$, and $\mathrm{GSH}+$ pretreated groups were calculated to be $12.12,7.92$, and $4.65 \mu \mathrm{M}$ $\mathrm{mL}^{-1}$ at $72 \mathrm{~h}$. The NGP/DOX and NGG/DOX groups pretreated by GSH had the lowest $\mathrm{IC}_{50}$, demonstrating their enhanced suppressor capability against the tumor cells and their advantage as potential antitumor drug formulations.

\section{In vivo Antitumor Efficacy}

The most important indicators of drug use in vivo were efficacy and safety, which must be rigorously evaluated before any newly formulations could be used clinically. Tumor-grafted animal models are the main method to evaluate the antitumor activities of drug delivery systems. As shown, compared with free DOX. $\mathrm{HCl}$, DOX-loaded nanogels exhibited stronger antitumor effect (Figure 4A). This result was related to two factors: one was related to the EPR effect (Huang et al., 2015; Shi et al., 2017), which resulted more DOX-loaded nanogels accumulated in tumor sites, the other was due to the more controlled release of DOX from nanogels, which resulted less DOX was released in circulation and more DOX accumulated in tumor sites. The tumor inhibition rates of NGP/DOX/3, NGP/DOX/6, $\mathrm{NGG} / \mathrm{DOX} / 3$, and $\mathrm{NGG} / \mathrm{DOX} / 6$ groups were $98.9 \pm 0.2 \%$, $99.4 \pm 0.03 \%, 97.15 \pm 0.4 \%$, and $99.1 \pm 0.1 \%$, which were much stronger than that of DOX/3 and DOX/6 groups (i.e., $87.4 \pm 2.1 \%$ and $95.8 \pm 3.0 \%$; $P<0.001$ ). Despite DOX also showed antitumor effect, the weight loss of mice was very obvious during the therapy (Figure 4B), especially in the DOX/6 group, indicating the severe systemic toxicity of DOX. In contrast, the groups treated with DOX-loaded nanogels showed tiny body weight loss, indicating the effective attenuation effect DOX-loaded nanogels. Furthermore, organ indices were provided to offer a general impression of the system toxicity of DOX. The tumor index was also calculated. As shown, the organ indices exhibited no obvious difference except the tumor indices (Figure 5), indicating these nanogels would efficiently suppress the tumor growth while not cause systemic toxicities in vivo.

In addition, the histopathological and immunohistochemical tests were performed to further confirm the antitumor efficacy of DOX-loaded nanogels (Figure 6). In this study, four immunohistochemical stainings caspase-3, survivin, Bax, and Bcl-2 were performed (Figure 6). It is well known that the process of cell apoptosis is regulated and controlled by various apoptotic genes. Notably, the caspase family plays a crucial role in the process of apoptosis (Huang et al., 2015; Shi et al., 2017). The activation of apoptosis-inducing factor caspase-3 is the key pathway for a variety of stimuliinduced apoptosis (Huang et al., 2015; Shi et al., 2017). As shown, the signals of pro-apoptotic protein Bax (brown) and caspase-3 (brown) of the nanogels treatment groups were much stronger than those of the free DOX. $\mathrm{HCl}$ treatment groups. In contrast, the anti-apoptotic protein Bcl-2 (brown)
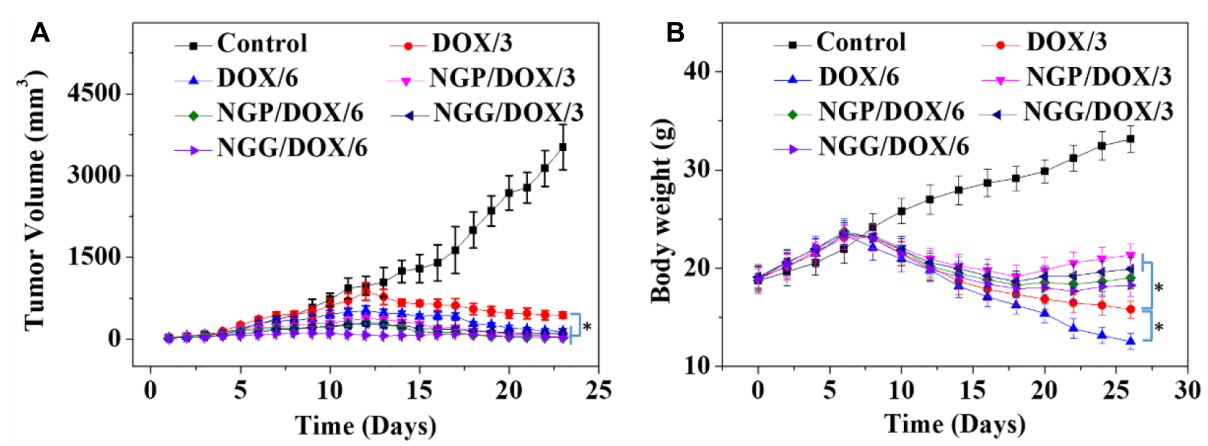

FIGURE 4 | Tumor volume (A) and body weight (B) changes of Lewis lung carcinoma-bearing BALB/c mice in the course of treatment with control, or free DOX.HCl, or NGP/DOX, or NGG/DOX at a dosage of 3.0 or $6.0 \mathrm{mg} \mathrm{DOX.HCl} \mathrm{equivalent} \mathrm{per} \mathrm{kg} \mathrm{body} \mathrm{weight.} \mathrm{Each} \mathrm{set} \mathrm{of} \mathrm{data} \mathrm{is} \mathrm{represented} \mathrm{as} \mathrm{mean} \pm S D(n=10$; $* P<0.001)$. 

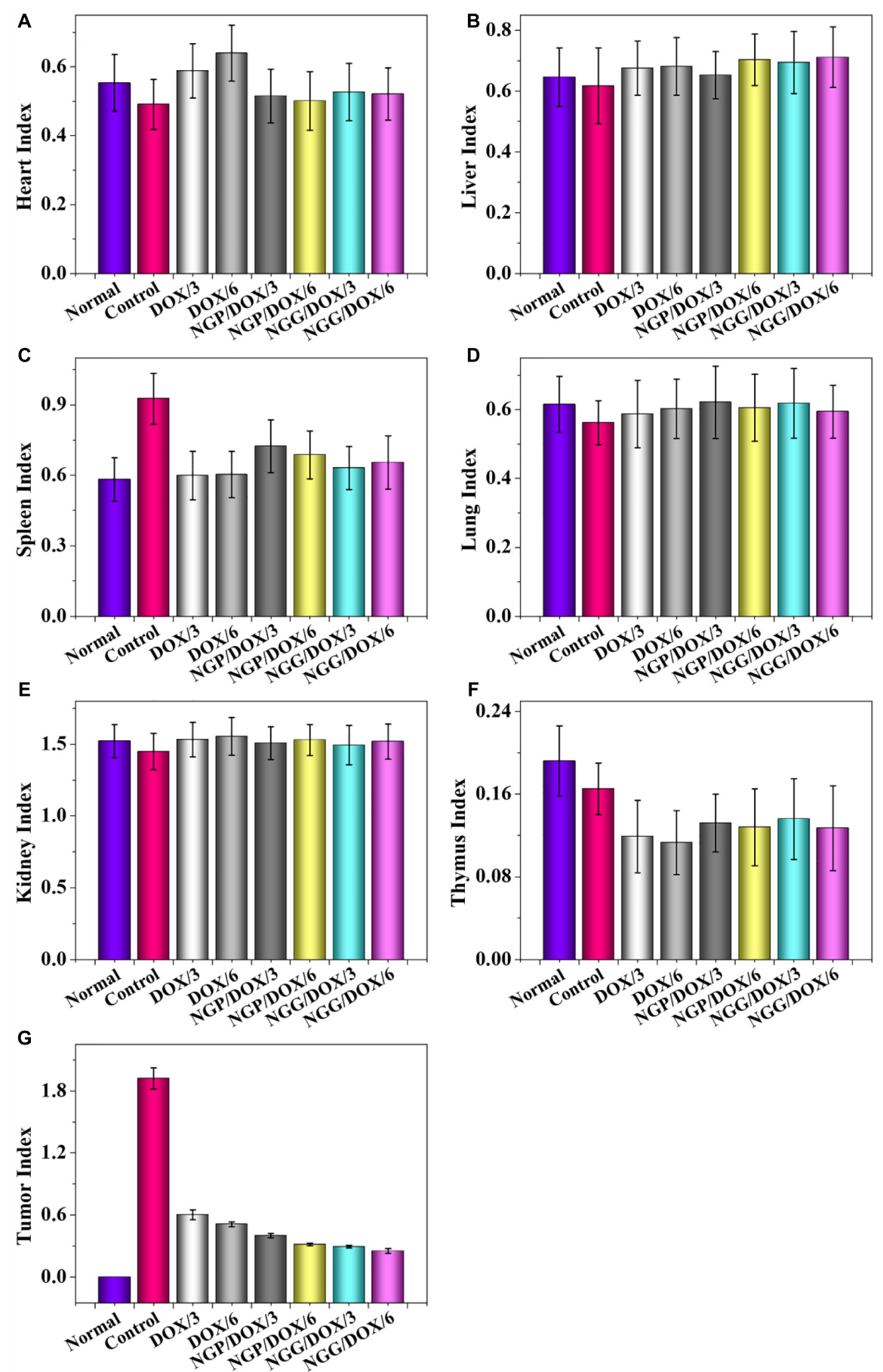

FIGURE $\mathbf{5}$ | Evaluations of heart (A), liver (B), spleen (C), lung (D), kidney (E), thymus (F), and tumor (G) indexes of Lewis lung carcinoma-allografted mice after injected with NS, DOX/3, DOX/6, NGP/DOX/3, NGP/DOX/6, NGG/DOX/3, or NGG/DOX/6. Each set of data is represented as mean $\pm S D(n=8)$.

showed an obvious decline in the nanogels treatment groups. Furthermore, survivin was also tested to evaluate the cell survival (Huang et al., 2015; Shi et al., 2017). As shown, the signals of survivin (brown) were obviously reduced in the DOXloaded nanogels treatment groups. These results fully verified that our DOX-loaded nanogels, especially NGP/DOX/6 and NGG/DOX/6, could be served as efficient nano-therapeutic agents.

As shown in Figure 6, H\&E staining showed universal mitosis and mild hemorrhagic necrosis in the control 


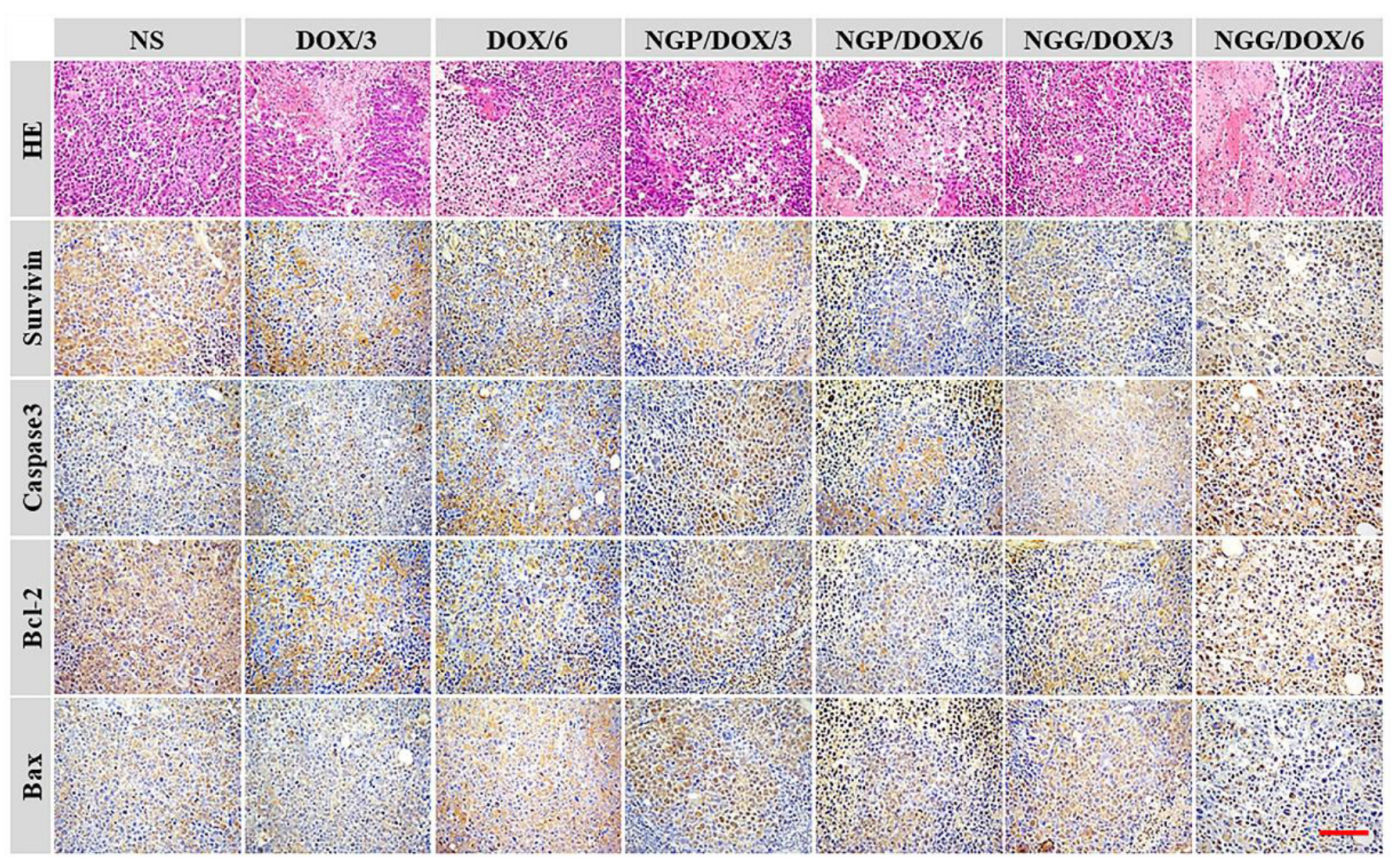

FIGURE 6 | Immunohistochemical (i.e., caspase-3, survivin, Bax, and Bcl-2) analyses of tumor tissue sections after treatments with NS, free DOX.HCl at a dose of 3 and $6 \mathrm{mg}(\mathrm{DOX} / 3, \mathrm{DOX} / 6)$, NGP/DOX, and NGG/DOX at a dose of 3.0 or $6.0 \mathrm{mg}$ DOX.HCl equivalent per kg body weight. Scale bar = 50 $\mu \mathrm{m}$, magnification: $200 \times$.

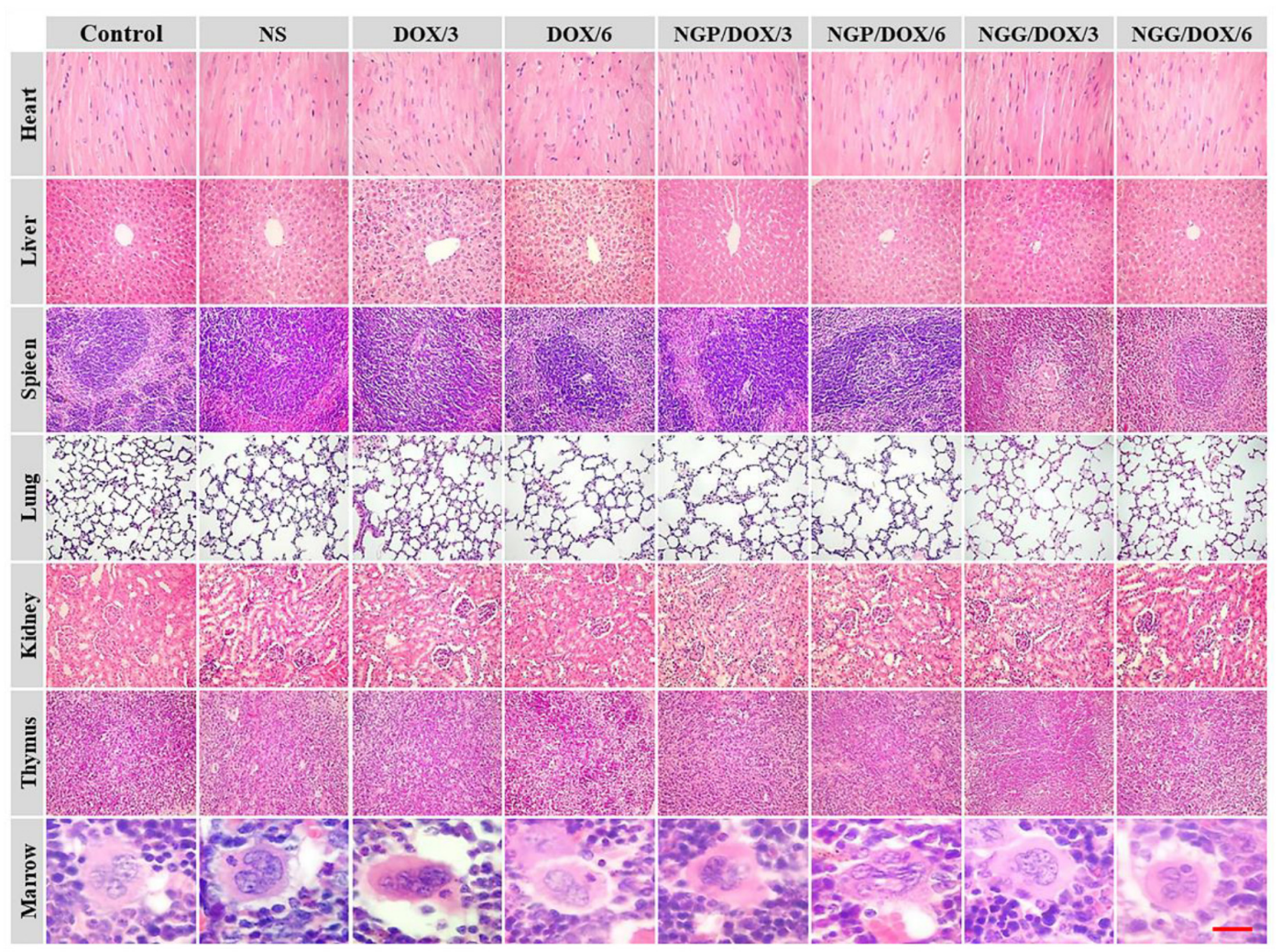

FIGURE 7 | EX vivo histopathological analyses (i.e., H\&E) of lung tumor, visceral organ, thymus, and marrow sections after all treatments with control, NS, free DOX 3 and $6 \mathrm{mg}(\mathrm{DOX} / 3, \mathrm{DOX} / 6)$, NGP/DOX, and NGG/DOX at a dose of 3.0 or $6.0 \mathrm{mg} \mathrm{DOX.HCl} \mathrm{equivalent} \mathrm{per} \mathrm{kg} \mathrm{body} \mathrm{weight} \mathrm{(NG/DOX/3,} \mathrm{NG/DOX/6).} \mathrm{Magnification:}$ $200 \times$ 

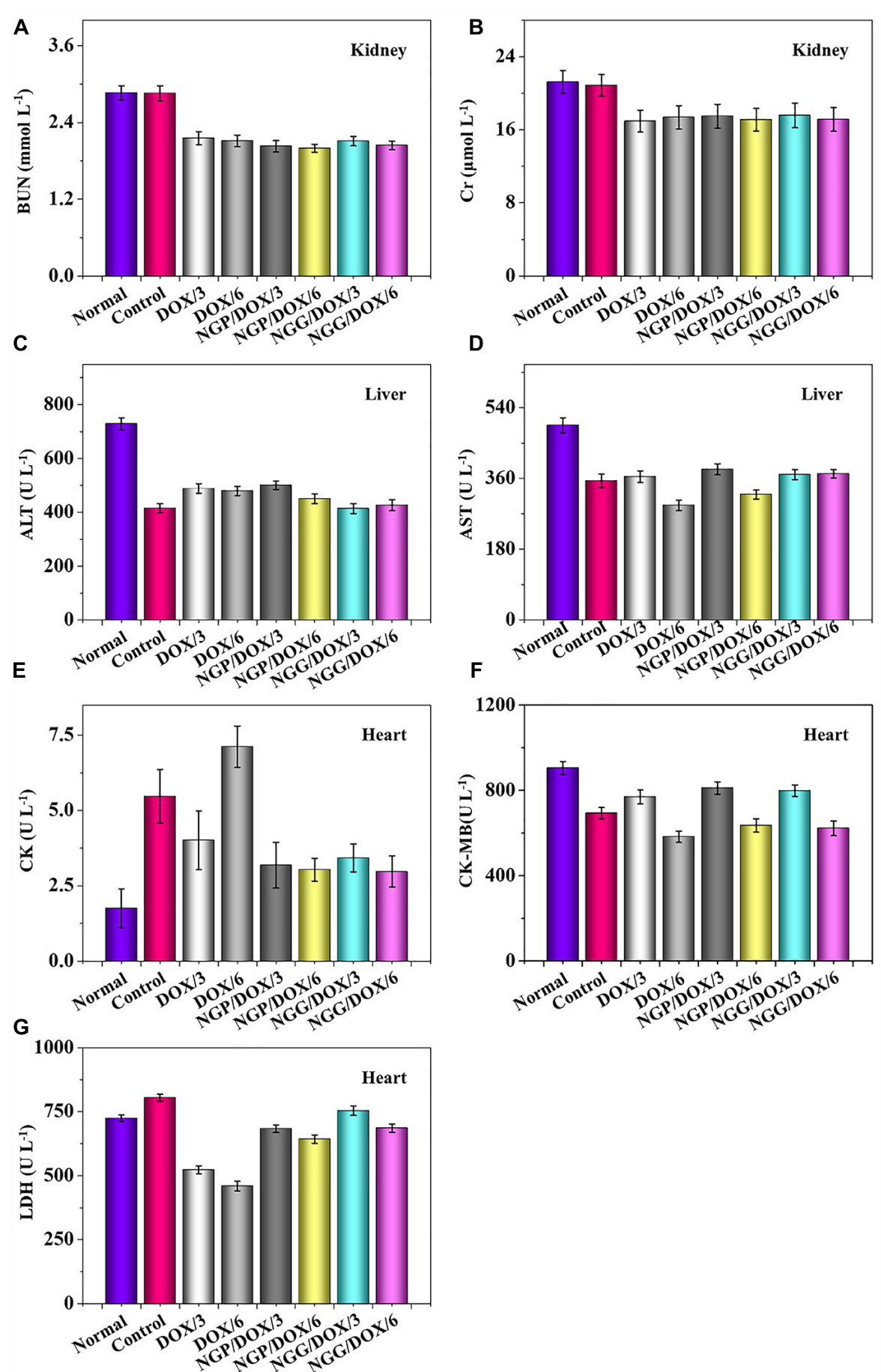

FIGURE 8 | Biochemical parameter assays for safety evaluation. Evaluations of BUN (A), Cr (B), ALT (C), AST (D), CK (E), CK-MB (F), and LDH (G) in corresponding internal organs of normal mice or Lewis lung carcinoma-allografted BALB/c mice after treatment with NS, free DOX.HCl at a dose of 3 and 6 mg (DOX/3, DOX/6), NGP/DOX, and NGG/DOX at a dose of 3.0 or $6.0 \mathrm{mg}$ DOX.HCl equivalent per $\mathrm{kg}$ body weight. Each set of data is represented as mean $\pm S D(n=3)$.

group, indicating the rapid cell growth. In contrast, all the DOX formulations treatment groups showed varying degrees of tumor growth suppression. Specifically, DOX formulations caused a reduction in mitosis and extensive hemorrhage and necrosis. The treatment groups were ranked as follows according to the relative amount of necrotic tissues: NGP/DOX/6.0 > NGG/DOX/6.0 > DOX/6.0 > NGP/DOX/3.0 > NGG/DOX/3.0 > DOX/3.0. Furthermore, the results of semiquantitative in Figures 10C,F showed the necrotic areas of NGP/DOX/6 and NGP/DOX/6 treatment groups were 1.4 and 

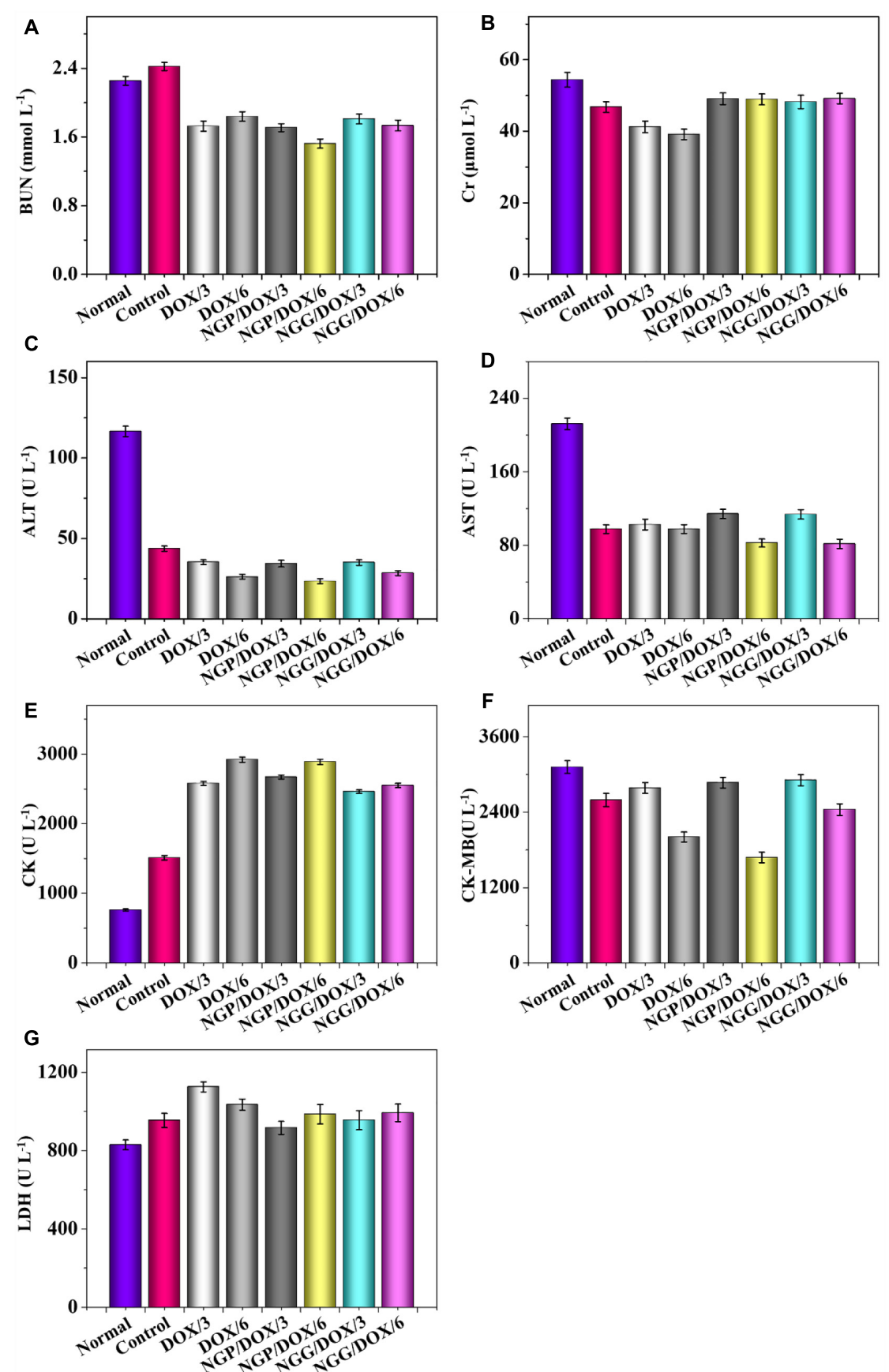

FIGURE 9 | Biochemical parameter assays for safety evaluation. Evaluations of BUN (A), Cr (B), ALT (C), AST (D), CK (E), CK-MB (F), and LDH (G) in serum of normal mice or Lewis lung carcinoma-allografted BALB/c mice after treatment with NS, free DOX.HCl at a dose of 3 and 6 mg (DOX/3, DOX/6), NGP/DOX, and $\mathrm{NGG} / \mathrm{DOX}$ at a dose of 3.0 or $6.0 \mathrm{mg} \mathrm{DOX} \cdot \mathrm{HCl}$ equivalent per $\mathrm{kg}$ body weight. Each set of data is represented as mean $\pm S D(n=3)$.

1.2 times larger than those of the free $\mathrm{DOX} \cdot \mathrm{HCl} / 6$ treatment group, respectively.

\section{In vivo Security Evaluation}

In this study, systematic safety was evaluated by monitoring the physical conditions and body weights changes, by analyzing the pathological morphology of various organs, by detecting the biological parameters from organs and serum, and by examining the BMMR and WBC levels after therapeutics. The in vivo systematic toxicity of DOX was reflected by body weight and histopathology of organs. Similar body weight gain trends were observed in each group of nude mice within the initial 1-10 days (Figure 4B). After that, the body weights of the nanogels treatment groups still showed similar 

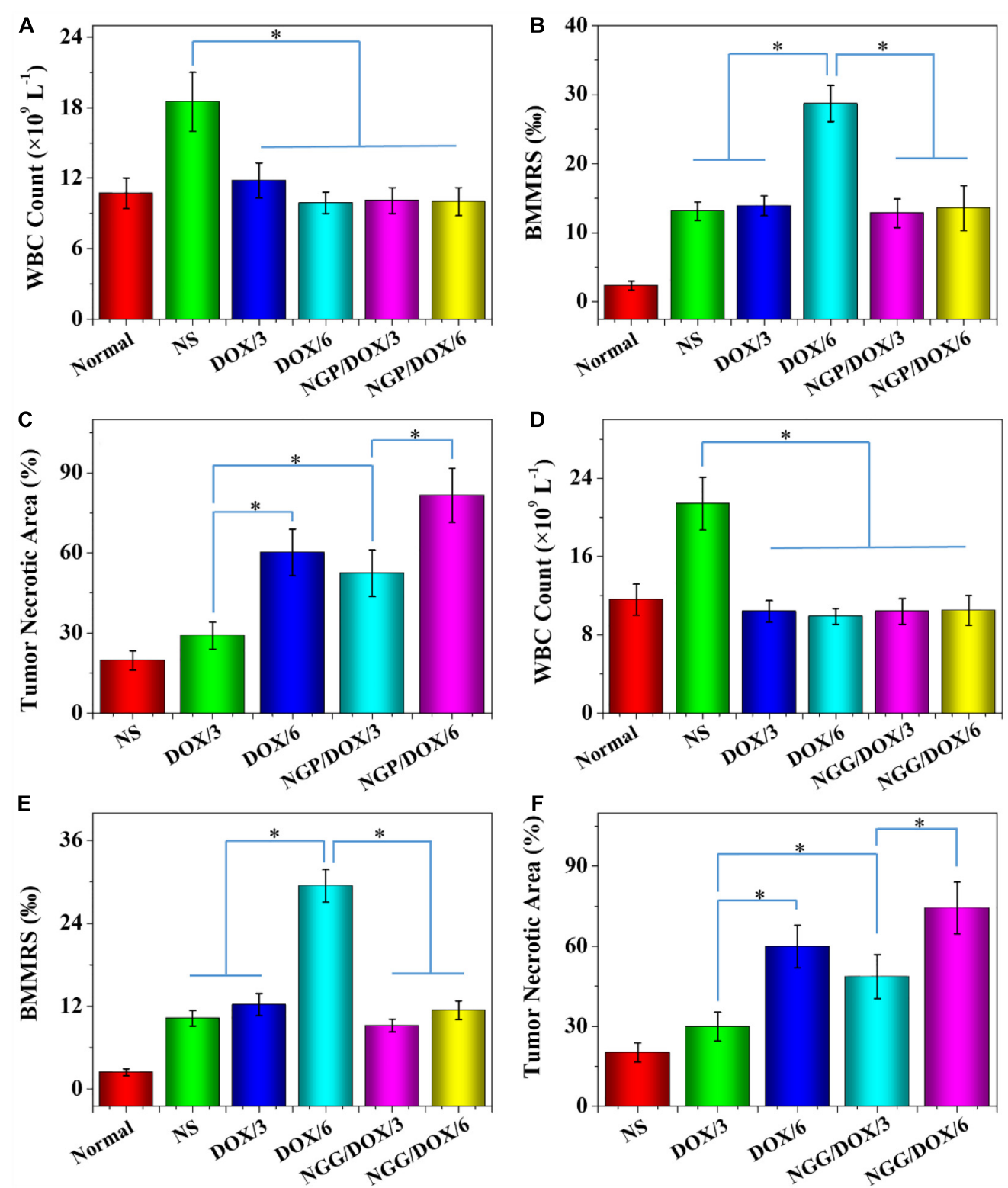

FIGURE 10 | WBC counts (A,D), BMMRs (B,E) and tumor necrotic area from H\&E (C,F), normal mice (as control), or Lewis lung carcinoma-allografted BALB/C nude mice after treatment with NS, or free DOX.HCl or NGP/DOX (A-C) or NGG/DOX (D-F) at a dose of 3.0 or 6.0 mg DOX.HCl equivalent per kg body weight. Normal BALB/c nude mice serve as controls (Normal). Each set of data is represented as mean $\pm \operatorname{SD}(n=10 ; * P<0.001)$.

growth trends on day 11-26. This result might be related to that controlled release of nanogels, which resulted less DOX release in the circulation. On the contrary, the body weights of free $\mathrm{DOX} \cdot \mathrm{HCl}$ treated groups showed significant downward trends within the same time interval, and larger doses cause more weight loss $(P<0.001)$. Especially the dose of $6.0 \mathrm{mg}(\mathrm{kg} \mathrm{BW})^{-1}$ DOX treatment group caused severe weight loss, indicating the toxicity of DOX were dosedependent.

The histopathological analyses of major organs were shown in Figure 7. Significant neutrophil accumulation and myocardial fiber breakage were observed in the showed in the free DOX. $\mathrm{HCl}$ treatment groups. In contrast, the neutrophil accumulation did not occur in the nanogels treatment groups, the myocardial cells were arranged orderly, and the sarcolemma-maintained integrity, probably relate to the reduced accumulation of free $\mathrm{DOX} \cdot \mathrm{HCl}$ in heart. Moreover, the microregional necrosis of hepatocytes in the free DOX $\cdot \mathrm{HCl}$ treatment group indicated that free $\mathrm{DOX} \cdot \mathrm{HCl}$ had significant hepatotoxicity. On the contrary, less structural interferences were observed in the nanogels treatment groups. Moreover, the nephrotoxicity of free $\mathrm{DOX} \cdot \mathrm{HCl}$ was also reduced by the nanogels, which was verified by the intact structure of the kidneys in the nanogels treatment groups. These results indicated that the DOX-loaded nanogels could effectively reduce 
systematic toxicity, probably due to the satisfactory stability of DOX-loaded nanogels. All the results confirmed that the DOXloaded nanogels had good biocompatibility.

The relevant clinical parameters of heart (CK, CK-MB, and $\mathrm{LDH}$ ), liver (ALT and AST), and kidney (BUN and Cr) can reflect the function of corresponding organs. In this work, these clinical parameters were detected to verify the safety of DOXloaded nanogels in vivo. As shown, the parameters of all organs except heart in each treated group were within the normal range (Figure 8). Free DOX $\cdot \mathrm{HCl} / 6$ could cause serious damage to the heart (Figure 8E). The relevant parameters in serum were also tested by the commercial ELISA kits. All the clinical parameters of mice treated with DOX-loaded nanogels were equal or lower than normal nude mice treated with NS except CK (Figure 9). These results indicated that DOX-loaded nanogels did not cause serious organ dysfunction.

The number of WBC was tested to reflect the influence of chemotherapy drugs on the immune system. As shown in Figures 10A,D, the WBC counts of the NS treatment group was increased obviously. On the contrary, the DOX formulation treatment groups did not show increased WBC counts. The results indicated that the treatments with DOX formulations could effectively reduce the inflammation induced by tumor $(P<0.01)$.

The genotoxicity caused by chemotherapy drugs can be quantified by BMMR (Wang et al., 2018). As shown in Figures 10B,E, H\&E-stained marrow sections were used to observe bone marrow mononuclear cells. These histopathological sections were also used to calculate BMMRs. Compared with normal mice, the BMMRs increased obviously in Lewis lunggrafted mice. For the mice treated with DOX formulations,

\section{REFERENCES}

Acharya, S., and Sahoo, S. K. (2011). PLGA nanoparticles containing various anticancer agents and tumour delivery by EPR effect. Adv. Drug Deliv. Rev. 63, 170-183. doi: 10.1016/j.addr.2010.10.008

Allen, T. M., and Cullis, P. R. (2013). Liposomal drug delivery systems: from concept to clinical applications. Adv. Drug Deliv. Rev. 65, 36-48. doi: 10.1016/j. addr.2012.09.037

Armstrong, J. P. K., and Stevens, M. M. (2018). Strategic design of extracellular vesicle drug delivery systems. Adv. Drug Deliv. Rev. 130, 12-16. doi: 10.1016/j. addr.2018.06.017

Chen, J., Ding, J., Wang, Y., Cheng, J., Ji, S., Zhuang, X., et al. (2017a). Sequentially responsive shell-stacked nanoparticles for deep penetration into solid tumors. Adv. Mater. 29:1701170. doi: 10.1002/adma.201701170

Chen, J., Ding, J., Xu, W., Sun, T., Xiao, H., Zhuang, X., et al. (2017b). Receptor and microenvironment dual-recognizable nanogel for targeted chemotherapy of highly metastatic malignancy. Nano Lett. 17, 4526-4533. doi: 10.1021/acs. nanolett.7b02129

Cheng, R., Meng, F., Deng, C., Klok, H.-A., and Zhong, Z. (2013). Dual and multistimuli responsive polymeric nanoparticles for programmed site-specific drug delivery. Biomaterials 34, 3647-3657. doi: 10.1016/j.biomaterials.2013.01.084

Cong, Y., Xiao, H., Xiong, H., Wang, Z., Ding, J., Li, C., et al. (2018). Dual drug backboned shattering polymeric theranostic nanomedicine for synergistic eradication of patient-derived lung cancer. Adv. Mater. 30:1706220. doi: 10. 1002/adma.201706220

Ding, J., Chen, L., Xiao, C., Chen, L., Zhuang, X., and Chen, X. (2014). Noncovalent interaction-assisted polymeric micelles for controlled drug delivery. Chem. Commun. 50, 11274-11290. doi: 10.1039/C4CC03153A a dose-related increase in BMMR was observed. Moreover, the BMMRs of the groups treated with free $\mathrm{DOX} \cdot \mathrm{HCl}$ were significantly greater than those of groups treated with DOXloaded nanogels $(P<0.001)$. These results demonstrated that the physiological damage caused by free DOX was dose-dependent. Fortunately, DOX-loaded nanogels could effectively mitigate this injury.

\section{CONCLUSION}

In this work, reduction-responsive DOX-loaded nanogels (NGP/DOX and NGG/DOX) were prepared by classical nanoprecipitation method. In vitro studies showed that both NGP/DOX and NGG/DOX groups exhibited stronger cellular uptake of Lewis cells compared with free DOX treatment groups. Furthermore, all the NGP/DOX and NGG/DOX groups exhibited more efficient antitumor efficacy than the free DOX $\cdot \mathrm{HCl}$ treatment groups in the Lewis lung carcinoma grafted nude mouse model. Importantly, all the DOX-loaded nanogels could significantly reduce the systemic toxicity of DOX. Therefore, these polypeptides nanogels with high systemic safety could serve as promising nanodrug delivery platforms for the future lung carcinoma chemotherapy.

\section{AUTHOR CONTRIBUTIONS}

All authors conceived and designed the study. KN, NL, and YY performed the experiments. KN, NL, YY, CG, YG, and JW analyzed and interpreted the data, and prepared the manuscript.

Ding, J., Li, C., Zhang, Y., Xu, W., Wang, J., and Chen, X. (2015). Chiralitymediated polypeptide micelles for regulated drug delivery. Acta Biomater. 11, 346-355. doi: 10.1016/j.actbio.2014.09.043

Ding, J., Shi, F., Xiao, C., Lin, L., Chen, L., He, C., et al. (2011). One-step preparation of reduction-responsive poly(ethylene glycol)-poly (amino acid)s nanogels as efficient intracellular drug delivery platforms. Polym. Chem. 2, 2857-2864. doi: $10.1039 / \mathrm{clpy} 00360 \mathrm{~g}$

Ding, J., Shi, F., Xiao, C., Zhuang, X., He, C., and Chen, X. (2013a). Facile preparation of $\mathrm{pH}$ and reduction responsive PEGylated polypeptide nanogel for efficient doxorubicin loading and intracellular delivery. J. Control. Release 172, E40-E41. doi: 10.1016/j.jconrel.2013. 08.087

Ding, J., Xiao, C., Li, Y., Cheng, Y., Wang, N., He, C., et al. (2013b). Efficacious hepatoma-targeted nanomedicine self-assembled from galactopeptide and doxorubicin driven by two-stage physical interactions. J. Control. Release 169, 193-203. doi: 10.1016/j.jconrel.2012.12.006

Ding, J., Xu, W., Zhang, Y., Sun, D., Xiao, C., Liu, D., et al. (2013c). Self-reinforced endocytoses of smart polypeptide nanogels for "on-demand" drug delivery. J. Control. Release 172, 444-455. doi: 10.1016/j.jconrel.2013.05.029

Feng, X.-R., Ding, J.-X., Gref, R., and Chen, X.-S. (2017). Poly( $\beta$-cyclodextrin)mediated polylactide-cholesterol stereocomplex micelles for controlled drug delivery. Chin. J. Polym. Sci. 35, 693-699. doi: 10.1007/s10118-017-1932-7

Ge, Z., and Liu, S. (2013). Functional block copolymer assemblies responsive to tumor and intracellular microenvironments for site-specific drug delivery and enhanced imaging performance. Chem. Soc. Rev. 42, 7289-7325. doi: 10.1039/ c3cs60048c

Guo, H., Li, F., Xu, W., Chen, J., Hou, Y., Wang, C., et al. (2018). Mucoadhesive cationic polypeptide nanogel with enhanced penetration for 
efficient intravesical chemotherapy of bladder cancer. Adv. Sci. 5:1800004. doi: 10.1002/advs.201800004

Guo, H., Xu, W., Chen, J., Yan, L., Ding, J., Hou, Y., et al. (2017). Positively charged polypeptide nanogel enhances mucoadhesion and penetrability of 10hydroxycamptothecin in orthotopic bladder carcinoma. J. Control. Release 259, 136-148. doi: 10.1016/j.jconrel.2016.12.041

Huang, K., Shi, B., Xu, W., Ding, J., Yang, Y., Liu, H., et al. (2015). Reductionresponsive polypeptide nanogel delivers antitumor drug for improved efficacy and safety. Acta biomater. 27, 179-193. doi: 10.1016/j.actbio.2015.08.049

Ji, X. Y., Kong, N., Wang, J. Q., Li, W. L., Xiao, Y. L., Gan, S. T., et al. (2018). A novel top-down synthesis of ultrathin 2D boron nanosheets for multimodal imaging-guided cancer therapy. Adv. Mater. 30:e1803031. doi: 10.1002/adma. 201803031

Jiang, Z., Chen, J., Cui, L., Zhuang, X., Ding, J., and Chen, X. (2018a). Advances in stimuli-responsive polypeptide nanogels. Small Methods 2:1700307. doi: 10 . 1002/smtd.201700307

Jiang, Z. Y., Chen, J. J., Cui, L. G., Zhuang, X. L., Ding, J. X., and Chen, X. S. (2018b). Advances in stimuli-responsive polypeptide nanogels. Small Methods 2:1700307. doi: 10.1002/smtd.201700307

Kanodra, N. M., Silvestri, G. A., and Tanner, N. T. (2015). Screening and early detection efforts in lung cancer. Cancer 121, 1347-1356. doi: 10.1002/cncr. 29222

Kernstine, K. (2017). "Treatment of small cell lung neoplasms by surgery," in Modern.Thoracic Oncology, eds R. B. Cameron, O. Olevsky, and D. Gage (Singapore: World Scientific Publishing Company), 189-196.

Kosakowska, K. A., Casey, B. K., Kurtz, S. L., Lawson, L. B., and Grayson, S. M. (2018). Evaluation of amphiphilic star/linear-dendritic polymer reverse micelles for transdermal drug delivery: directing carrier properties by tailoring core versus peripheral branching. Biomacromolecules 19, 3163-3176. doi: 10. 1021/acs.biomac.8b00680

Li, D., Feng, X., Chen, L., Ding, J., and Chen, X. (2018a). One-step synthesis of targeted acid-labile polysaccharide prodrug for efficiently intracellular drug delivery. ACS Biomater. Sci. Eng. 4, 539-546. doi: 10.1021/acsbiomaterials. $7 \mathrm{~b} 00856$

Li, J., Xu, W., Li, D., Liu, T., Zhang, Y. S., Ding, J., et al. (2018b). A locally deployable nanofiber patch for sequential drug delivery in treatment of primary and advanced orthotopic hepatomas. ACS Nano 12, 6685-6699. doi: 10.1021/ acsnano.8b01729

Li, S., Dong, S., Xu, W., Tu, S., Yan, L., Zhao, C., et al. (2018c). Antibacterial hydrogels. Adv. Sci. 5:1700527. doi: 10.1002/advs.201700527

Li, S., Zhang, T., Xu, W., Ding, J., Yin, F., Xu, J., et al. (2018d). Sarcoma-targeting peptide-decorated polypeptide nanogel intracellularly delivers shikonin for upregulated osteosarcoma necroptosis and diminished pulmonary metastasis. Theranostics 8, 1361-1375. doi: 10.7150/thno.18299

Liu, X., Wang, J., Xu, W., Ding, J., Shi, B., Huang, K., et al. (2015). Glutathionedegradable drug-loaded nanogel effectively and securely suppresses hepatoma in mouse model. Int. J. Nanomed. 10, 6587-6602. doi: 10.2147/IJN.S90000

Mura, S., Nicolas, J., and Couvreur, P. (2013). Stimuli-responsive nanocarriers for drug delivery. Nat. Mater. 12, 991-1003. doi: 10.1038/nmat3776

Parks, S. K., Chiche, J., and Pouysségur, J. (2013). Disrupting proton dynamics and energy metabolism for cancer therapy. Nat. Rev. Cancer 13, 611-623. doi: $10.1038 / \mathrm{nrc} 3579$

Phillips, D. J., and Gibson, M. I. (2014). Redox-sensitive materials for drug delivery: targeting the correct intracellular environment, tuning release rates, and appropriate predictive systems. Antioxid. Redox Signal. 21, 786-803. doi: $10.1089 /$ ars. 2013.5728

Piffoux, M., Silva, A. K. A., Wilhelm, C., Gazeau, F., and Tareste, D. (2018). Modification of extracellular vesicles by fusion with liposomes for the design of personalized biogenic drug delivery systems. ACS Nano 12, 6830-6842. doi: 10.1021/acsnano.8b02053

Ryu, J.-H., Jiwpanich, S., Chacko, R., Bickerton, S., and Thayumanavan, S. (2010). Surface-functionalizable polymer nanogels with facile hydrophobic guest encapsulation capabilities. J. Am. Chem. Soc. 132, 8246-8247. doi: 10. 1021/ja102316a

Shen, K., Li, D., Guan, J., Ding, J., Wang, Z., Gu, J., et al. (2017). Targeted sustained delivery of antineoplastic agent with multicomponent polylactide stereocomplex micelle. Nanomed. Nanotechnol. Biol. Med. 13, 1279-1288. doi: $10.1016 /$ j.nano.2016.12.022
Shi, B., Huang, K., Ding, J., Xu, W., Yang, Y., Liu, H., et al. (2017). Intracellularly swollen polypeptide nanogel assists hepatoma chemotherapy. Theranostics 7 , 703-716. doi: 10.7150/thno.16794

Sun, X., Wang, G., Zhang, H., Hu, S., Liu, X., Tang, J., et al. (2018). The blood clearance kinetics and pathway of polymeric micelles in cancer drug delivery. ACS Nano 12, 6179-6192. doi: 10.1021/acsnano.8b02830

Tao, W., Zhang, J., Zeng, X., Liu, D., Liu, G., Zhu, X., et al. (2015). Blended nanoparticle system based on miscible structurally similar polymers: a safe, simple, targeted, and surprisingly high efficiency vehicle for cancer therapy. Adv. Healthcare Mater. 4, 1203-1214. doi: 10.1002/adhm.201400751

Tao, W., Zhu, X. B., Yu, X. H., Zeng, X. W., Xiao, Q. L., Zhang, X. D., et al. (2017). Black phosphorus nanosheets as a robust delivery platform for cancer theranostics. Adv. Mater. 29:1603276. doi: 10.1002/adma.201603276

Wang, J., Xu, W., Li, S., Qiu, H., Li, Z., Wang, C., et al. (2018). Polylactidecholesterol stereocomplex micelle encapsulating chemotherapeutic agent for improved antitumor efficacy and safety. J. Biomed. Nanotechnol. 14, 2102-2113. doi: $10.1166 /$ jbn.2018.2624

Xiao, H., Yan, L., Dempsey, E. M., Song, W., Qi, R., Li, W., et al. (2018). Recent progress in polymer-based platinum drug delivery systems. Prog. Polym. Sci. 87, 70-106. doi: 10.7497/j.issn.2095-3941.2015.0063

Xu, W., Ding, J., and Chen, X. (2017). Reduction-responsive polypeptide micelles for intracellular delivery of antineoplastic agent. Biomacromolecules 18, 32913301. doi: 10.1021/acs.biomac.7b00950

Yang, N., Ding, Y., Zhang, Y., Wang, B., Zhao, X., Cheng, K., et al. (2018). Surface functionalization of polymeric nanoparticles with umbilical cordderived mesenchymal stem cell membrane for tumor-targeted therapy. ACS Appl. Mater. Interfaces 10, 22963-22973. doi: 10.1021/acsami.8b0 5363

Ye, Y., Yu, J., and Gu, Z. (2016). Versatile protein nanogels prepared by in situ polymerization. Macromol. Chem. Phys. 217, 333-343. doi: 10.1002/macp. 201500296

Yu, J., Zhang, Y., Sun, W., Wang, C., Ranson, D., Ye, Y., et al. (2016). Endosomemimicking nanogels for targeted drug delivery. Nanoscale 8, 9178-9184. doi: 10.1039/C5NR08895J

Zha, L. S., Banik, B., and Alexis, F. (2011). Stimulus responsive nanogels for drug delivery. Soft Matter 7, 5908-5916. doi: 10.1039/c0sm01307b

Zhang, X., Liang, X., Gu, J., Chang, D., Zhang, J., Chen, Z., et al. (2017). Investigation and intervention of autophagy to guide cancer treatment with nanogels. Nanoscale 9, 150-163. doi: 10.1039/c6nr07866d

Zhang, Y., Cai, L., Li, D., Lao, Y.-H., Liu, D., Li, M., et al. (2018). Tumor microenvironment-responsive hyaluronate-calcium carbonate hybrid nanoparticle enables effective chemotherapy for primary and advanced osteosarcomas. Nano Res. 11, 4806-4822. doi: 10.1007/s12274-018-2066-0

Zhao, K., Li, D., Xu, W., Ding, J., Jiang, W., Li, M., et al. (2017). Targeted hydroxyethyl starch prodrug for inhibiting the growth and metastasis of prostate cancer. Biomaterials 116, 82-94. doi: 10.1016/j.biomaterials.2016.11. 030

Zhao, Y., Alakhova, D. Y., Kim, J. O., Bronich, T. K., and Kabanov, A. V. (2013). A simple way to enhance Doxil (R) therapy: drug release from liposomes at the tumor site by amphiphilic block copolymer. J. Control. Release 168, 61-69. doi: 10.1016/j.jconrel.2013. 02.026

Zhu, Q., Chen, X., Xu, X., Zhang, Y., Zhang, C., and Mo, R. (2018). Drug delivery: tumor-specific self-degradable nanogels as potential carriers for systemic delivery of anticancer proteins (Adv. Funct. Mater. 17/2018). Adv. Func. Mat. 28:1870109. doi: $10.1002 /$ adfm.201870109

Conflict of Interest Statement: The authors declare that the research was conducted in the absence of any commercial or financial relationships that could be construed as a potential conflict of interest.

Copyright $\odot 2019 \mathrm{Niu}, \mathrm{Li}$, Yao, Guo, Ge and Wang. This is an open-access article distributed under the terms of the Creative Commons Attribution License (CC BY). The use, distribution or reproduction in other forums is permitted, provided the original author(s) and the copyright owner(s) are credited and that the original publication in this journal is cited, in accordance with accepted academic practice. No use, distribution or reproduction is permitted which does not comply with these terms. 